\title{
ON STEADY TRANSONIC FLOW BY COMPENSATED COMPACTNESS
}

\author{
Cathleen S. Morawetz
}

\section{Introduction}

This paper contains a theorem for the mixed equations of potential flow in two space variables that is analogous to DiPerna's theorem [2] on the existence of weak solutions for two hyperbolic conservation laws and is based on the Tartar-Murat Lemma for compensated compactness, see [6]. The application is plane flow for which a suitable "viscous" model exists, and this will be discussed in another paper. Some hypothesis about speed must be made. There are other conceivable applications such as axiallysymmetric flow, plane fluid models for semiconductors, etc. The equations of the viscous model must admit a potential and a stream function, or something like it. This is crucial in proving that the limit at zero viscosity is a genuine weak solution. But also one has to establish some underlying bounds.

The difficult problem in the applications is to create a problem depending on a "viscous" parameter and which has certain properties: It must be solvable and have sufficiently strong bounds uniform in the viscous parameter $\nu$ so that the theorem given here can be applied.

Here we are assuming we have such a viscous model. In Section 2 , we describe the system that will be considered. In Section 3, we find entropy pairs of a simple form that satisfy divergence estimates in the potential-stream function plane. In Section 4, we justify using this plane and describe the use of the Tartar-Murat Lemma and the Young measure for the weak limits. We also state the theorem to be proved. In Sections 5, 6, and 7, we show that, under the circumstances of the theorem, we can divide the proof into three parts each with its own applications of the entropy pairs. The hyperbolic region is treated by a variation of Serre's method, see [5].

This approach to transonic flow was first presented in [3]. However, the application erred. The entropy pairs used in extending DiPerna's hyperbolic results were not shown to be bounded in the elliptic region as well as in the hyperbolic region. Here explicit, bounded, entropy pairs are found by separation of variables and achieve the desired result.

\section{Transonic potential system}

By a transonic potential system we mean a system for the velocity $(u, v)$ and density $\rho_{B}$ of the form

$$
\begin{gathered}
u_{y}-v_{x}=0, \\
\left(\rho_{B} u\right)_{x}+\left(\rho_{B} v\right)_{y}=0, \\
\rho_{B}=\rho_{B}(q),
\end{gathered}
$$

Received April 14, 1994, revised November 15, 1994.

1991 Mathematics Subject Classification. 75-xx, 76-xx, 76N10, 76N15, 76H05, 35M05, 35L-xx.

Key words and phrases: fluid dynamics, compressible transonic flow, mixed equations, compensated compactness. 
where $q^{2}=u^{2}+v^{2}$ and $\rho_{B} q d q+c^{2}(\rho) d \rho_{B}=0$.

The first equation is the condition for potential flow and implies $(u, v)=\nabla \phi$. The third is Bernoulli's law which comes from Euler's momentum equations. The density $\rho_{B}$ given by Bernoulli's law is assumed to satisfy the condition that $\rho_{B} q$ is a convex function with a maximum at sonic speed and vanishing for $q=0$ and $q=q^{*}$ when $\rho_{B}=0$. The second equation is conservation of mass and implies the existence of a stream function $\nabla \psi=(-\rho v, \rho u)$.

Remark 2.1. Other variations, for future consideration, occur in axially symmetric transonic flow where (2.2) is replaced by $\left(y \rho_{B} u\right)_{x}+\left(y \rho_{B} v\right)_{y}$ and $\nabla \psi=\left(-y \rho_{B} v, y \rho_{B} u\right)$. However, for these cases no viscous models have been constructed. Another variation is, in (2.3), to let $\rho$ depend on $\phi$ and possibly related functions.

We limit ourselves here to the plane case and first consider some limited aspects of a viscous model and associated assumptions. Let $\nu$ be the viscous parameter and let

$$
\begin{gathered}
\left(u^{\nu}, v^{\nu}\right)=\nabla \phi^{\nu}, \\
\rho_{B}=\rho_{B}\left(q^{\nu}\right), \quad\left(q^{\nu}\right)^{2}=\left(u^{\nu}\right)^{2}+\left(v^{\nu}\right)^{2} .
\end{gathered}
$$

Let $u^{\nu}, v^{\nu}$ approximately satisfy the mass law

$$
\left(\rho_{B}\left(q^{\nu}\right) u^{\nu}\right)_{x}+\left(\rho_{B}\left(q^{\nu}\right) v^{\nu}\right)_{y}=e,
$$

where $e \rightarrow 0$ as $\nu \rightarrow 0$ and is compact in $H_{\text {loc }}^{-1}$ for $\nu<\nu_{0}$, say.

In addition, we require a viscous stream function that approximates the real one for some $R^{\nu}$ :

$$
\left(-R^{\nu} v^{\nu}, R^{\nu} u^{\nu}\right)=\nabla \psi^{\nu}
$$

Our objective is to use the DiPerna-Tartar-Murat approach with a family of entropy pairs to show that if $R^{\nu} \rightarrow \rho_{B}\left(q^{\nu}\right)$ in some appropriate sense, then there exists a subsequence of $\nu$ such that $u^{\nu}, v^{\nu}$ converge to a weak solution of (2.1)-(2.3) almost everywhere.

\section{Entropy pairs}

In this section we introduce bounded entropy pairs $(f, g)$ that are generated by $(2.1)-(2.3)$. Here $(f, g)$ is a vector function of the variables $(u, v)$. In the standard DiPerna-Tartar-Murat method, we would be led to require estimates on $\operatorname{div}(f, g)$ and as a result conclude, by using families of entropy vectors, that there exists a subsequence of the parameter $\nu$ such that the corresponding viscous solutions converge to a weak solution of equations (2.1)-(2.3). However, it is not clear how to find a family of entropy vectors satisfying $\operatorname{div}(f, g)=0$ formally that will permit us to draw the right conclusions in the $x, y$-plane. In this section, we drop the subscript $B$ on $\rho$ and proceed formally.

We introduce the stream function and the potential by

$$
\nabla \phi=(u, v), \quad \nabla \psi=(-\rho v, \rho u),
$$

where $\rho$ is given by Bernoulli's law as a function of $u^{2}+v^{2}$.

We may rewrite the equations as

$$
\operatorname{div} \rho \nabla \phi=0, \quad \operatorname{div} \tau \nabla \psi=0,
$$

where

$$
\tau=\rho^{-1} \text {. }
$$


Then we regard $(f, g)$ as a vector function of $\phi, \psi$ where we note that the Jacobian of the transformation from $\phi, \psi$ to $x, y$ coordinates is $\rho\left(u^{2}+v^{2}\right)$ which vanishes only at cavitation $\rho=0$ or stagnation $u^{2}+v^{2}=0$. Since one of our assumptions will be that $u^{2}+v^{2}$ and $\rho$ are bounded uniformly away from 0 , we may use the two planes equivalently in this section. More care must be exercised in examining weak limits.

We generate entropy pairs using

$$
\operatorname{div}(f \rho \nabla \phi+g \tau \nabla \psi)=0
$$

or, by (3.2),

$$
f_{\phi}+g_{\psi}=0
$$

Of course, we can construct entropy pairs $F, G$ with $\operatorname{div}(F, G)=0$ using $F=f \rho u-g v$, $G=f \rho v+g u$, but they do not appear to be so useful.

It is helpful to represent (3.1) by

$$
\begin{gathered}
d \phi+i \tau d \psi=w d z, \\
w=e^{\sigma-i \theta}, \quad d z=d(x+i y) .
\end{gathered}
$$

A computation using (3.6) readily shows that

$$
\begin{gathered}
\rho \sigma_{\psi}-\theta_{\phi}=0 \\
\tau \sigma_{\phi}+\theta_{\psi}=\tau_{\phi}=\dot{\tau} \sigma_{\phi}
\end{gathered}
$$

where $\dot{\tau}$ is $\frac{d \tau}{d \sigma}$ here. Then, from (3.5) and (3.7), we have, with $f=f(\sigma, \theta), g=g(\sigma, \theta)$,

$$
f_{\sigma} \sigma_{\phi}+f_{\theta} \rho \sigma_{\psi}+g_{\sigma} \sigma_{\psi}+g_{\theta}(\dot{\tau}-\tau) \sigma_{\phi}=0
$$

so that we require

$$
\begin{gathered}
f_{\sigma}+g_{\theta}(\dot{\tau}-\tau)=0 \\
f_{\theta}+g_{\sigma} \tau=0
\end{gathered}
$$

Then (3.5) holds.

We shall use only certain solutions of (3.9). First, we introduce

$$
d \mu=\rho d \sigma \quad \text { with } \quad \mu=0
$$

for sonic speed, i.e., $e^{2 \sigma}=c^{2}(\rho)$. From (2.2),

$$
\frac{1}{2} \cdot e^{2 \sigma}+i(\rho)=\text { const. }
$$

and $e^{2 \sigma} d \sigma+\tau c^{2} d \rho=0$, so $\dot{\tau}$ in (3.9) is $\tau e^{2 \sigma} / c^{2}=\tau M^{2}$ with $M$ the Mach number. Thus, (3.9) may be written as

$$
\begin{gathered}
f_{\mu}+g_{\theta} \tau^{2}\left(M^{2}-1\right)=0 \\
f_{\theta}+g_{\mu}=0
\end{gathered}
$$

Any solution of (3.12) may be found by setting

$$
\begin{aligned}
& f=H_{\mu}, \\
& g=-H_{\theta},
\end{aligned}
$$

if $H$ is a solution of

$$
\tau^{2}\left(M^{2}-1\right) H_{\theta \theta}-H_{\mu \mu}=0,
$$

where $M^{2}-1=0$ for $\mu=0$. This equation is of Tricomi type. 
The only entropy pairs that we shall use here are those obtained by separation of variables. Thus, with ${ }^{\cdot}$ denoting $d / d \mu$,

$$
f=e^{ \pm i n \theta} \dot{H}_{ \pm n}, \quad g=e^{ \pm i n \theta}(\mp i n) H_{ \pm n},
$$

where $H_{ \pm n}$ satisfies the ordinary differential equation

$$
\ddot{H}_{ \pm n}-n^{2} \tau^{2}\left(1-M^{2}\right) H_{ \pm n}=0,
$$

and

$$
f=e^{ \pm n \theta} \dot{K}_{ \pm n}, \quad g=e^{ \pm n \theta}(\mp n) K_{ \pm n},
$$

where

$$
\ddot{K}_{ \pm n}-n^{2} \tau^{2}\left(M^{2}-1\right) K_{ \pm n}=0 .
$$

Such solutions exist in both elliptic and hyperbolic regions.

\section{Use of the Tartar-Murat Lemma on compensated compactness}

We first formulate conditions on a sequence of solutions of the regularized equation, i.e., (2.1), (2.4), (2.5).

Suppose the solutions $u, v$ of the regularized equations have a weakly convergent subsequence which satisfy uniformly

(i) $-\infty<\mu_{1}<\mu<\mu_{2}<\mu_{*}$,

(ii) $\theta_{1} \leq \theta \leq \theta_{2}$,

where $\mu_{*}$ is the value of $\mu$ at cavitation speed, and

(iii) $F_{x}+G_{y}$ is compact in $H_{\text {loc }}^{-1}$ for all bounded smooth entropy pairs $F, G$.

Then every smooth function of $\mu, \theta$ also has a weakly convergent subsequence.

As in [6], we may represent the weak limit (w. 1.) of any function $\widetilde{F}(\mu, \theta)$ by a Young measure, $d \tilde{\nu}(\mu, \theta, x, y)$,

$$
\text { w. 1. } \tilde{F}=\int \tilde{F}(\mu, \theta) d \tilde{\nu}(\mu, \theta, x, y),
$$

where the integral is over the $\mu, \theta$ domain. Because of (iii), we are able to claim that the Tartar-Murat Lemma holds.

Tartar-Murat Lemma. If $\left(F_{1}, G_{1}\right)$ and $\left(F_{2}, G_{2}\right)$ are two pairs of entropy functions satisfying (iii), then

$$
\text { w. l. } F_{1} \text { w. l. } G_{2}-\text { w. l. } G_{1} \text { w. l. } F_{2}=\int\left(F_{1}(\mu, \theta) G_{2}(\mu, \theta)-G_{1}(\mu, \theta) F_{2}(\mu, \theta)\right) d \tilde{\nu}
$$

where the integral is over the domain given by (i) and (ii).

Note that over the same domain, we see that by taking $\tilde{F} \equiv 1$,

$$
\int d \tilde{\nu}=1 .
$$

The object is, by using a suitable infinite sequence of entropy pairs, to show that the Young measure is a Dirac measure at a single point, say $\theta_{0}, \mu_{0}$. We then have

$$
\text { w. l. } \tilde{F}(\mu, \theta) \text { at } x, y \text { is } \tilde{F}\left(\mu_{0}(x, y), \theta_{0}(x, y)\right) \text { a.e. }
$$

From (4.4) it follows that the weak limit of an appropriate subsequence of solutions of (2.1), (2.2), (2.4) exists and for all $\chi \subset C_{0}^{\infty}$,

$$
\int\left(\chi_{x} \rho_{B} u+\chi_{y} \rho_{B} v\right) d x d y=0
$$


so that $u, v$ are genuine weak solutions of (2.1)-(2.3).

However, it turns out to be necessary to work in the $\phi, \psi$ plane where the entropy pairs $(f, g)$ are of such a simple form that the Tartar-Murat Lemma leads to a Dirac measure. Since $\phi, \psi$ depend on $\nu$, this may appear confusing, and our object now is to justify this step.

Suppose $\chi(\phi, \psi) \subset C_{0}^{\infty}$ in a compact region $\Omega$ of the $\phi, \psi$ space. Suppose for each $\nu, u$ and $v$ are bounded functions of $x, y$. Then they are bounded functions of $\phi, \psi$ and $\phi, \psi$ have uniformly bounded derivatives with respect to $x, y$. And under the assumption that $\rho_{B}$ and $u^{2}+v^{2}$ are bounded away from zero, $x, y$ have uniformly bounded derivatives with respect to $\phi, \psi$ for all $\nu$.

In order to use the Tartar-Murat Lemma on open sets $\Omega$ in the $\phi, \psi$ plane, we see first that $\int_{\Omega} \chi(\phi, \psi)\left(\begin{array}{l}u \\ v\end{array}\right) d \phi d \psi$ contains a subsequence that converges since $\left(\begin{array}{l}u \\ v\end{array}\right)$ is uniformly bounded. Hence we may introduce a Young measure $d \nu(\theta, \mu, \phi, \psi)$ and then

$$
\underset{\phi, \psi}{\mathrm{w} .} \mathrm{l} . g(\theta, \mu)=\int g(\theta, \mu) d \nu(\theta, \mu, \phi, \psi) \text {. }
$$

Here $\theta, \mu$ are given by (3.6) and (3.12) and satisfy (i) and (ii).

Suppose, as we will show, that the measure is a Dirac measure. Then

$$
\underset{\phi, \psi}{\text { w. } l .} g(\theta, \mu)=g\left(\theta_{0}, \mu_{0}\right)
$$

where $\theta_{0}, \mu_{0}$ depend on $\phi, \psi$.

It remains to show that the weak limit with respect to $\phi, \psi$ is the weak limit with respect to $x, y$. But $\underset{\phi, \psi}{\mathrm{w} .} . g(\theta, \mu)$ satisfies

$$
\lim \int \chi(\phi, \psi) g(\theta, \mu) d \phi d \psi=\int \chi(\phi, \psi) \underset{\phi, \psi}{\mathrm{w} .} \operatorname{l} . g(\theta, \mu) d \phi d \psi
$$

And the left-hand side is

$$
\begin{aligned}
& \lim \int \chi(\phi(x, y), \psi(x, y)) g(\theta, \mu) \rho_{B}\left(u^{2}+v^{2}\right) d x d y \\
= & \lim \int \chi\left(\phi^{\nu}(x, y) \psi^{\nu}(x, y)\right) g\left(\theta^{\nu}, \mu^{\nu}\right) R^{\nu}\left(u^{2}+v^{2}\right)^{\nu} d x d y
\end{aligned}
$$

for a subsequence since $R^{\nu} \rightarrow \rho_{B}$.

By an appropriate subsequence, this limit differs arbitrarily little, by the uniform continuity of $\phi^{\nu}, \psi^{\nu}$, from

$$
\lim \int C(x, y) g\left(\theta^{\nu}, \mu^{\nu}\right) \rho^{\nu}\left(q^{\nu}\right)^{2} d x d y=\int C(x, y) \underset{x, y}{\mathrm{w} .} \operatorname{l.} g \rho\left(q^{2}\right) d x d y
$$

where $C$ is $C_{0}^{\infty}$. But the right-hand side is, by (4.6),

$$
\begin{gathered}
\int \chi(\phi(x, y), \psi(x, y)) g\left(\theta_{0}(\phi, \psi), \mu_{0}(\phi, \psi)\right) d \phi d \psi \\
=\int \chi(\phi(x, y) \psi(x, y)) g \rho\left(u^{2}+v^{2}\right) d x d y
\end{gathered}
$$

where $g \rho\left(u^{2}+v^{2}\right)$ is evaluated at $\theta_{0}, \mu_{0}$ which are functions of $\phi(x, y), \psi(x, y)$. Here $\phi$ and $\psi$ are the limit solutions as $\nu \rightarrow 0$ which are continuous functions of $x, y$.

This reduces the weak existence theory to establishing the estimates (i), (ii), and (instead of (iii))

(iv) $f_{\phi}+g_{\psi}$ is compact in $H_{\text {loc }}^{-1}$ where the domain is an open set in the $\phi, \psi$ plane for $f, g$ smooth bounded functions of $\theta, \mu, \nu$.

We conclude 
Lemma 4.1. If $f, g$ satisfy (iv) and are bounded, the Tartar-Murat Lemma holds with a Young measure $d \tilde{\nu}(\mu, \theta ; \psi, \phi)$.

The theorem we shall prove is

Theorem 4.2. Suppose (i) $\theta^{*}, \mu^{*}$ are the weak limits for some subsequence of solutions of

$$
\begin{gathered}
u_{y}^{\nu}-v_{x}^{\nu}=0, \quad\left(R^{\nu} u^{\nu}\right)_{x}+\left(R^{\nu} v^{\nu}\right)_{y}=0, \\
\left(\rho_{B} u^{\nu}\right)_{x}+\left(\rho_{B} v^{\nu}\right)_{y} \subset H_{l o c}^{-1}, \quad \rho_{B}=\rho_{B}\left(\mu^{\nu}\right),
\end{gathered}
$$

where $u-i v=e^{\sigma-i \theta}, \sigma=\sigma(\mu)$ by (3.12). Suppose the subsequences $\mu\left(u^{\nu}, V^{\nu}\right)$ and $\theta\left(u^{\nu}, v^{\nu}\right)$ satisfy the inequality in (iii) below.

(ii) $(f, g)$ is any bounded smooth entropy pair satisfying

$$
f_{\phi}+g_{\psi} \subset H_{\text {loc }}^{-1} \text { where } \nabla \phi=\left(u^{\nu}, v^{\nu}\right)
$$

and $\nabla \psi=\left(-R^{\nu} v^{\nu}, R^{\nu} u^{\nu}\right)$ where $R^{\nu} \rightarrow \rho_{B}\left(\mu^{\nu}\right)$ as $\nu \rightarrow 0$.

(iii) $(f, g)$ is bounded in

$$
-\infty<-\mu_{1}<\mu<\mu_{2}, \quad|\theta|<B,
$$

where $\mu_{2}$ is less than its cavitation value.

Then the weak limits $\left(\theta^{*}, \mu^{*}\right)$ are strong almost everywhere and every function $g(\theta, \mu)$ converges a.e. to $g\left(\theta^{*}, \mu^{*}\right)$.

The proof consists of showing successively that the Young measure $d \tilde{\nu}$ (for the weak limit in $\phi, \psi$ space $)$ in the $(\theta, \mu)$ domain is

(a) Dirac or zero in the elliptic region;

(b) Dirac in the elliptic region or confined to a characteristic quadrilateral in the hyperbolic region with possibly one parabolic point;

(c) if in the hyperbolic-parabolic region, then confined to two characteristics of opposite kind and hence to a point.

The proofs of (a), (b), (c) are the contents of Sections 5, 6, and 7. The conclusion is that the Young measure is Dirac under the conditions of the theorem and, hence, the limit of the sequence is a weak solution of the nonlinear equations (2.1),(2.2).

\section{The elliptic domain}

In this section, we show that the Young measure is a Dirac measure in the elliptic region or has its support in the hyperbolic-parabolic subdomain of $\mathcal{D}$ defined by (i), (ii) of Section 4. The proof here simplifies that of [3].

We generate the two pairs using, see (3.17)-(3.18),

$$
\begin{gathered}
f_{1}=\dot{H}_{n} e^{i n \theta}, \quad g_{1}=-i n H_{n} e^{+i n \theta}, \\
f_{2}=\dot{H}_{n} e^{-i n \theta}, \quad g_{2}=+i n H_{n} e^{-i n \theta},
\end{gathered}
$$

where $H_{n}$ will be chosen as the solution of $\ddot{H}_{n}+n^{2} \tau^{2}\left(M^{2}-1\right) H_{n}=0$ which grows exponentially as $\mu \rightarrow-\infty$ where $M \rightarrow 0$ and $\tau \rightarrow$ const. Later, we will describe $H_{n}$ more completely.

By the Tartar-Murat Lemma in the $\phi, \psi$ plane, see [6],

$$
\iint\left(f_{1} g_{2}-f_{2} g_{1}\right) d \tilde{\nu}=\int f_{1} d \tilde{\nu} \int g_{2} d \tilde{\nu}-\int f_{2} d \tilde{\nu} \int g_{1} d \tilde{\nu}
$$


Since the Young measure $d \tilde{\nu}$ is a probability measure so that $\int d \tilde{\nu}=1$, we may rewrite this equation as

$$
\iint\left(f_{1}\left(g_{2}-g_{2}^{\prime}\right)-f_{2}\left(g_{1}-g_{1}^{\prime}\right)\right) d \tilde{\nu} d \tilde{\nu}^{\prime}=0
$$

where ' denotes that the variables are $\theta^{\prime}, \mu^{\prime}$ and the domain of integration is over both primed and unprimed variables. We make use of the fact that we may interchange the primed and unprimed variables to obtain

$$
\iint\left(\left(f_{1}-f_{1}^{\prime}\right)\left(g_{2}-g_{2}^{\prime}\right)-\left(f_{2}-f_{2}^{\prime}\right)\left(g_{1}-g_{1}^{\prime}\right)\right) d \tilde{\nu} d \tilde{\nu}^{\prime}=0
$$

We now substitute (5.1) and find, as in [3],

$$
\begin{aligned}
0=\iint( & \left(H_{n}(\mu)-H_{n}\left(\mu^{\prime}\right)\right)\left(\dot{H}_{n}(\mu)-\dot{H}_{n}\left(\mu^{\prime}\right)\right) \\
& \left.+4 H_{n}(\mu) \dot{H}_{n}\left(\mu^{\prime}\right) \sin ^{2} \frac{1}{2} n\left(\theta-\theta^{\prime}\right)\right) d \tilde{\nu} d \tilde{\nu}^{\prime} .
\end{aligned}
$$

Suppose the measure has an elliptic part $\mu<0$ and a hyperbolic part $\mu>0$. We look at the integrand for just two of the values, an unprimed "elliptic" and a primed "hyperbolic". And suppose $n$ is large. Then the primed contribution will be highly oscillatory but bounded and the unprimed contribution coming from $H_{n}(\mu) \dot{H}_{n}(\mu)$ will grow like $\exp \left(-2 n \int \tau \sqrt{1-M^{2}} d \mu\right)$. Here $H_{n}$ is a solution of the ordinary differential equation normalized to 1 at $\mu=0$ where it is also flat. In the hyperbolic region, it oscillates but is bounded; for $\mu<0$, it grows exponentially in $n$ or as $\mu \rightarrow-\infty$. Near $\mu=0$, its asymptotics are more involved. So it will dominate everything else in the integral unless $\int d \tilde{\nu}^{\prime}$ is zero. We rigorize this argument now. We use Lemma 4 from [7].

Lemma 5.1 (Lemma 4 of [7]). Let $\dot{p}=-\tilde{\tau}\left(1-M^{2}\right)^{1 / 2}, p(0)=0, \frac{1}{2}(-\dot{p})^{-1 / 2}=h(\mu)$.

(i) For $0 \leq \mu \leq \mu\left(\sigma_{+}\right), H_{n}, n^{-1} \dot{H}_{n}$ are bounded independently of $n$ if $\sigma_{+}<s_{*}$.

(ii) There exist functions $B(\delta)$ bounded in $\delta$ and $C_{n}(\delta)>h^{2}(-\delta)$ such that

(a) for $\mu_{-} \leq \mu \leq 0$,

$$
\begin{gathered}
1<H_{n}(\mu)<B(\delta) e^{n\left(p\left(\mu_{-}\right)-p(-\delta)\right)}, \\
0<-n^{-1} \dot{H}_{n}(\mu)<B(\delta) e^{n\left(p\left(\mu_{-}\right)-p(-\delta)\right)}
\end{gathered}
$$

(b) for $\mu_{-} \leq \mu \leq-2 \delta, n>N_{0}(\delta)$,

$$
\begin{gathered}
H_{n}(\mu)>\frac{1}{4} C_{n}(\delta) h(-\delta) h(\mu) e^{n(p(\mu)-p(-\delta))}, \\
-n^{-1} \dot{H}_{n}(\mu)>\frac{1}{4} C_{n}(\delta) h(-\delta) h^{3}(\mu) e^{n(p(\mu)-p(-\delta))} .
\end{gathered}
$$

From (iia) and the inequality (iii) of Theorem 4.2, we see that the whole integrand of (5.5) with both points elliptic is nonpositive. Here we use both $-\dot{H}_{n}>0$ and $\ddot{H}_{n}=n^{2} \tau^{2}\left(1-M^{2}\right) H_{n}>0$.

From (i) we see that the integrand is bounded by $n$ with both points hyperbolic.

From (iib) we see that if one point is elliptic and the other hyperbolic or parabolic the contribution from the elliptic term $H_{n} \dot{H}_{n}$ behaves like $-n e^{2 n(p(\mu)-p(-\delta))}$ for $|\delta| \ll|\mu|$. Such terms dominate. Thus, either there is measure only in the parabolichyperbolic region or the measure is entirely supported in the parabolic-elliptic domain. But in the latter case, as we observed above, the integrand is nonpositive and vanishes only if the measure is concentrated at a point. Hence, we conclude 
Lemma 5.2. The measure $d \tilde{\nu}(\theta, \mu, \phi, \psi)$ is either supported in the parabolic-hyperbolic subdomain or it is a Dirac measure in the elliptic region.

\section{The hyperbolic domain reduced}

In this section, we will show that if the support of the measure $\nu$ is in the hyperbolic domain, then it is confined to a characteristic quadrilateral.

In [3] the entropy pairs for hyperbolic regions were obtained without noting that they must be bounded in the original $(\theta, \mu)$-rectangle in order to apply the TartarMurat Lemma. For this reason, they cannot be constructed from hyperbolic data as solutions of the partial differential equation (3.16) since they may not be continuable into the elliptic region. However, the entropy pairs $K_{ \pm n} e^{ \pm n \theta}$ can be continued since $K_{ \pm n}$ satisfies a regular ordinary differential equation in the elliptic region. We begin with Lemma 5 of [3] along with the identity corresponding to (5.5).

Thus we have

$\int\left(K_{-n}-K_{-n}^{\prime}\right)\left(\dot{K}_{-n}-\dot{K}_{-n}^{\prime}\right) d \tilde{\nu} d \tilde{\nu}^{\prime}=4 \int K_{-n} \dot{K}_{-n}^{\prime} \sinh ^{2} \frac{1}{2} n\left(\theta-\theta^{\prime}\right) d \tilde{\nu} d \tilde{\nu}^{\prime}$,

where, because of the results of Section 5 above, we now may assume there is no measure in the elliptic region. We also may replace $K_{-n}$ by $K_{+n}$ in (6.1). But we must specify them through a modification of a basic lemma about Airy functions.

Lemma 6.1. There exists a pair of linearly independent solutions $G_{ \pm n}(m)$ of the Airy equation $\ddot{G}-n^{2} m G=0$ which have the following properties:

(a) For $0 \leq m<n^{-2 / 3} x_{0}, G_{n}, n^{-2 / 3} \dot{G}_{n}, G_{-n}, n^{-1 / 3} \dot{G}_{-n}$ are bounded and for $n$ large enough $G_{-n}=1-\epsilon$ where $\epsilon \rightarrow 0$ as $x_{0} \rightarrow 0$.

(b) For $m \geq n^{-2 / 3} x_{0}$, the matrix

$$
\left(\begin{array}{cc}
G_{n} & G_{-n} \\
n^{-1} \dot{G}_{n} & n^{-1} \dot{G}_{-n}
\end{array}\right)
$$

has the asymptotic form

$$
\left(\begin{array}{cc}
e^{n \lambda} A_{n} & e^{-n \lambda} A_{-n} \\
e^{n \lambda}\left(\dot{\lambda} A_{n}+\dot{A}_{n}\right) & e^{-n \lambda}\left(-\dot{\lambda} A_{-n}+\dot{A}_{-n}\right)
\end{array}\right)
$$

where $A_{ \pm n}=m^{-1 / 4}+O\left(\frac{1}{n}\right), \lambda=\frac{2}{3} m^{3 / 2}$.

The fact that $K(\mu) \neq K^{\prime}(0) \mu$ leads to certain difficulties before we can apply this lemma. We use the methods of Wasow [7] or Bender and Orszag [1] and sketch the proof of the desired lemma which is exactly the same as for the Airy function since only the high-order terms are needed in the application.

The steps are

Lemma 6.2. There exists a change of variable from $\mu$ to $m(n, \mu)$ with the asymptotic form

$$
m=m_{0}(\mu)+\frac{1}{n^{2}} m_{1}(\mu) \cdots
$$

for large $n$, and from $P$ to $G$ with $P=h G$, where $h$ has the asymptotic form

$$
h \sim h_{0}(\mu)+\frac{1}{n^{2}} h_{1}(\mu),
$$

so that if $P$ satisfies

$$
\frac{d^{2} P}{d \mu^{2}}+n^{2} K(\mu) P=0, \quad K(\mu) \leq 0
$$


then

$$
\frac{d^{2} G}{d m^{2}}-n^{2} m G=0
$$

where $n$ is arbitrary.

The proof of this lemma follows simply by a formal if tedious substitution and expansion where we avoid the singularity of the differential equation at cavitation by using the assumption of the inequality of (iii) in Theorem 4.2.

The second step is to deduce from the Airy equation the leading terms of a $P$ that is analogous to $G_{ \pm}$. This is technical and we use only the fact that to lowest order we may take $m=-K(\mu)$ and use Lemma 6.1 to replace the two solutions for $P$, which we denote by $K_{ \pm n}$, by the two solutions $G_{ \pm n}$.

We substitute in the identity (6.1) using the second part of the lemma with $K_{-n} \sim e^{-n \lambda} m^{-1 / 4} \sim e^{-n \lambda}(\mu)^{-1 / 4}$ with $\lambda \sim \frac{2}{3} \mu^{3 / 2}$ for points where $m \geq n^{-2 / 3} x_{0}$, and by the first part of the Lemma we note that $K_{-n}$ and $n^{-2 / 3} \dot{K}_{-n}$ are bounded for $m \leq n^{-2 / 3} x_{0}$. Thus, we find that

$$
K_{-n} \dot{K}_{-n}^{\prime} \sim-n \dot{\lambda}^{\prime} e^{-n\left(\lambda+\lambda^{\prime}\right)} m^{-1 / 4}\left(m^{\prime}\right)^{-1 / 4}, \quad m \geq n^{-2 / 3} x_{0},
$$

or the integrand on the right-hand side behaves like

$$
-n \dot{\lambda}^{\prime} e^{-n\left(\lambda+\lambda^{\prime}\right)+n\left|\theta-\theta^{\prime}\right|} m^{-1 / 4} m^{\prime-1 / 4}
$$

while the left-hand side has a bounded integrand.

Thus, there can be no joint measure $d \tilde{\nu} d \tilde{\nu}^{\prime}$ in the domain where

$$
-\left(\lambda+\lambda^{\prime}\right)<\left|\theta-\theta^{\prime}\right|, \quad \lambda \text { or } \lambda^{\prime}>0 .
$$

Contributions from $\lambda=\lambda^{\prime}=0$ are all smaller. Hence, we conclude that there can be no measure in domains where

$$
-\left(\lambda+\lambda^{\prime}\right)<\left|\theta-\theta^{\prime}\right|, \quad \mu \text { or } \mu^{\prime} \neq 0 .
$$

Consider, without loss of generality, that $\theta>\theta^{\prime}$. Then there is no measure in $\theta+\lambda>\theta^{\prime}-\lambda^{\prime}$. The curves $\theta \pm \lambda=$ const. are the characteristics of the equation (3.16) for $H$. By looking at the two extremes we see that the g.l.b. $(\theta+\lambda)=$ l.u.b. $(\theta-\lambda)$ and, hence, there is no measure except in some wedge $\left(\theta-\theta_{0}\right)^{2}<\lambda^{2}$ or on the axis $\lambda=0$.

If there is measure, say, in the hyperbolic region and also on $\mu=0$, other than at $\theta=\theta_{0}$, say $\theta>\theta_{0}$, then there are points where $K_{-n} \dot{K}_{-n}^{\prime}\left(\sinh ^{2} n\left(\theta-\theta^{\prime}\right)\right) \sim$ $-n \dot{\lambda}^{\prime} e^{-n \lambda+n\left|\theta-\theta^{\prime}\right|} \dot{m}^{1 / 4}$ since $K_{-n} \sim 1$. But then $\left|\theta-\theta^{\prime}\right|<\lambda^{\prime}$, or

$$
\left(\theta-\left(\theta^{\prime}+\lambda^{\prime}\right)\right)\left(\theta-\left(\theta^{\prime}-\lambda^{\prime}\right)\right)<0,
$$

while $\left|\theta^{\prime} \pm \lambda^{\prime}\right|<\theta_{0}$, which is a contradiction. Thus, there is no measure on the parabolic axis for $\theta \neq \theta_{0}$ if there is measure in the hyperbolic region.

Suppose, to finish this section, that there is measure on the parabolic axis only. Then for any solution, say $\tilde{K}$, of $(3.18)$, we may choose to have $\widetilde{K}=1, n \dot{\widetilde{K}}=1$. The identity in $\widetilde{K}$ yields, since $\mu=\mu^{\prime}=0$ on the left-hand side,

$$
4 n^{-1} \int \sinh ^{2} \frac{n}{2}\left(\theta-\theta^{\prime}\right) d \tilde{\nu} d \tilde{\nu}^{\prime}=0
$$

from which it follows that the measure $d \nu$ is a Dirac measure. 
Thus, to summarize Sections 5 and 6:

- If the measure is not in the hyperbolic region, it is a Dirac measure at a point either on the parabolic line or in the elliptic region.

- If the measure is in the hyperbolic region, it lies between the characteristics $|\theta \pm \lambda|<\left|\theta_{0}\right|$ and there is no measure elsewhere.

Using the function $K_{+n}$, we note that since $\rho$ is bounded away from zero, the coefficient of $n$ is bounded and bounded away from zero. Thus we may use the same argument "upside down" and conclude that the support of the measure lies in $\left(\theta-\theta_{1}\right)^{2}<\left(\lambda-\lambda_{1}\right)^{2}$ where $\lambda_{1}$ is the maximum value of $\lambda_{1}$ given by the bound on the speed and $\theta_{1}$ is some value of $\theta$.

\section{The hyperbolic quadrilateral}

We have shown that the support of the Young measure is either entirely at a point in the elliptic region or on the parabolic line or else it lies in a quadrilateral bounded by characteristics in the hyperbolic region. We will now show that if it is the latter then the measure is a Dirac measure in either the hyperbolic region or on the parabolic line. The argument is based on

Lemma 7.1. If $\left(f_{i}, g_{i}\right), i=1,2,3$, are entropy pairs satisfying the assumptions of the Tartar-Murat Lemma and if $W_{i j}=\left|\begin{array}{ll}f_{i} & f_{j} \\ g_{i} & g_{j}\end{array}\right|$, then

$$
\left\langle f_{1}\right\rangle\left\langle W_{23}\right\rangle+\left\langle f_{2}\right\rangle\left\langle W_{31}\right\rangle+\left\langle f_{3}\right\rangle\left\langle W_{12}\right\rangle=0
$$

Here \langle\rangle means integrated with respect to the measure dĩ.

Note that a similar identity holds with $\left\langle g_{i}\right\rangle$ instead of $\left\langle f_{i}\right\rangle$.

Proof. Substitute from the Tartar-Murat Lemma

$$
\left\langle W_{23}\right\rangle=\left\langle f_{2}\right\rangle\left\langle g_{3}\right\rangle-\left\langle f_{3}\right\rangle\left\langle g_{2}\right\rangle,
$$

etc., and one sees that all terms cancel.

Serre [5] has used this identity in the hyperbolic problem for two entropy pairs that have no common support with a third, see also Morawetz [4]. This leaves only one term from which one can finally conclude by constructing Serre's special entropy pairs that the support of the measure lies on a single characteristic of one kind. Hence, it follows by using the same argument with characteristics of the other kind that the support lies at a point. His argument is simpler than DiPerna's. Here, because of the special form of the hodograph equations, we can simplify the argument and extend it to the mixed case. What follows also can be applied to any hyperbolic $2 \times 2$ case where there are suitable separated solutions of the hodograph equations.

Suppose $f, g$ are smooth entropy pairs satisfying $H_{\mu}=f,-H_{\theta}=g, K(\mu) H_{\theta \theta}+$ $H_{\mu \mu}=0$, and $\mu K(\mu) \leq 0, K^{\prime} \neq 0$. Let $H$ be of the form $P e^{n \theta}$ where $P$ is a solution of

$$
\ddot{P}+K n^{2} P=0 .
$$

We are going to use different entropy pairs of this form in the identity of the main lemma, but first we need a number of preliminary properties.

The three pairs of entropy functions that we use are found from $H_{1}=e^{-n \theta} G_{n}$, $H_{2}=e^{-(n+1) \theta} G_{n+1}$, and $H_{3}=e^{n \theta} G_{-n}$. They are chosen to mimic the behavior of pairs of compact support by yielding exponentially small contributions in the right places to the terms in the identity (7.1). 
We see from Lemma 6.1 that for $m \leq n^{-2 / 3} x_{0}, n^{-5 / 3} W_{12}$ is bounded and, for $m \geq n^{-2 / 3} x_{0}$, the leading term of the integrand of $W_{12}$ is

$$
e^{(2 n+1)(\lambda-\theta)}\left(A_{n} \dot{A}_{n+1}-\dot{A}_{n} A_{n+1}+\dot{\lambda} A_{n} A_{n+1}\right)
$$

or

$$
e^{(2 n+1)(\lambda-\theta)}\left(\left(\frac{2}{3} m^{e / 2}\right)^{\cdot} m^{-1 / 2}+O\left(\frac{1}{n}\right)\right) .
$$

Thus, for $\theta-\lambda<0$ as $n \rightarrow \infty, W_{12}$ is exponentially large with $n$.

On the other hand,

$$
W_{23}=e^{-\theta}\left|\begin{array}{cc}
-(n+1) G_{n+1} & n G_{-n} \\
\dot{G}_{n+1} & \dot{G}_{-n}
\end{array}\right|
$$

also is bounded by $n^{5 / 3}$ for $m<n^{-2 / 3} x_{0}$, and for $n$ large, $m \geq n^{-2 / 3} x_{0}$ using the asymptotics. Thus we find $n^{-2} W_{23}$ is bounded.

Similar bounds hold for $W_{31}$. On the other hand, $f_{1}=-n e^{-n \theta} G_{n}$ is bounded by $n e^{-n \theta}$ for $m<n^{-3 / 2} x_{0}$ and is asymptotically like $n e^{-n(\theta-\lambda)} m^{1 / 4}$ for $m \geq n^{-2 / 3} x_{0}$. Furthermore, $f_{2}=-(n+1) e^{-(n+1) \theta} G_{n+1}$ has similar behavior. Finally, $f_{3}=n e^{n \theta} G_{-n}$ is bounded from below by $n e^{n \theta}(1-\epsilon)$ for $m<n^{-3 / 2} x_{0}$ and behaves asymptotically like $n e^{n(\theta-\lambda)} m^{-1 / 4}$ for $m \geq n^{-3 / 2} x_{0}$. Pick $x_{0}$ small so that $\epsilon<1 / 2$.

We proceed to use these bounds in Lemma 7.1 to arrive at the conclusion that the domain of support of the measure is a single characteristic as in DiPerna [2] and Serre $[5]$.

Without loss of generality, suppose that the quadrilateral $-L \leq \theta-\lambda \leq 0$, $0 \leq \theta+\lambda \leq L$ is the smallest quadrilateral [in the sense that the values of the characteristic constants of the boundaries are either g.l.b. or l.u.b.] for which $\int d \tilde{\nu}=1$. We divide it by the characteristics $\theta-\lambda=-\delta, \theta-\lambda=-L+\delta$ with, say, $\delta=\frac{1}{10} L$.

We shall show that either the total measure in $-\delta \leq \theta-\lambda \leq 0$ is zero or the total measure between $-L \leq \theta-\lambda \leq-L+\delta$ is zero. Thus the quadrilateral is not the smallest quadrilateral unless the quadrilateral is a single characteristic segment.

We define $\left(\int g d \tilde{\nu}\right)_{\ell}$ as the integral of $g$ with respect to the measure contained in $-\ell \leq \theta-\lambda \leq 0$. From our estimates we find

$$
\int f_{3} d \tilde{\nu}>n\left(\int \chi(m) d \tilde{\nu}\right)_{\delta}
$$

where

$$
\begin{aligned}
& \chi=e^{-\delta}(1-\epsilon) \text { for } m<n^{-3 / 2} x_{0}, \\
& =e^{-\delta} m^{-1 / 4} \quad \text { for } \quad m \geq n^{-3 / 2} x_{0} .
\end{aligned}
$$

Also $\left|\int f_{1} d \tilde{\nu}\right|<b n e^{n L}$ and $\left|\int f_{2} d \tilde{\nu}\right|<b n e^{n L}$. Here $b$ is any bound independent of $n$.

On the other hand, $\int W_{12} d \tilde{\nu}=\left\langle W_{12}\right\rangle$ is greater than $c e^{(2 n+1)(L-\delta)} n(2 n+$ 1) $\left(\left(\int d \tilde{\nu}\right)_{L}-\left(\int d \tilde{\nu}\right)_{L-\delta}\right)$. Here $c$ is a constant greater than zero and independent of $n$. One also sees that $\left\langle f_{1}\right\rangle\left\langle W_{23}\right\rangle$ and $\left\langle f_{2}\right\rangle\left\langle W_{31}\right\rangle$ are bounded by $e^{n L} n^{2}$.

If we substitute these bounds in the identity of the main lemma and choose $n$ sufficiently large, we see that the term $\left\langle f_{3}\right\rangle\left\langle W_{12}\right\rangle$ dominates with the exponential $e^{(2 n+1)(L-\delta)-n \delta}$ over the other terms which are bounded by $e^{n L}$. Hence, we must have zero measure on the relevant domains. Thus, on substituting the above estimates, we have

$$
\left(\int d \tilde{\nu}\right)_{\delta}\left(\left(\int d \tilde{\nu}\right)_{L}-\left(\int d \tilde{\nu}\right)_{L-\delta}\right)=0
$$

which proves our assertion that the quadrilateral is not minimal. 
We conclude that the measure lies on a characteristic of one kind. By applying the argument reversing $\theta$, we conclude it lies on one of the other characteristics. Hence, it lies on a point, and since $\int d \tilde{\nu}=1$, we conclude that the measure is a Dirac measure.

Acknowledgement. I would like to acknowledge my debt to R. V. Kohn for many useful discussions and helpful contributions to this paper.

\section{References}

1. C. M. Bender and S. Orszag, Advanced Mathematical Methods for Scientists and Engineers, McGraw-Hill, New York, 1978, $118 f f$.

2. R. J. DiPerna, Convergence of approximate solutions to conservation laws, Arch. Rat. Mech. Anal. 82 (1983), 27-70.

3. C. S. Morawetz, On a weak solution for a transonic flow problem, CPAM 38 (1985), 797-818.

4. __ An alternate proof of DiPerna's theorem, CPAM 44 (1991), 1081-1090.

5. D. Serre, La compacité par compensation pour les systèmes hyperboliques nonlineaires de deux equations à une dimension d'espace, J. Math. Pures App. 65 (1986), 423-468.

6. L. C. Tartar, Compensated compactness and applications to partial differential equations, Nonlinear Analysis and Mechanics Vol. 39, Heriot-Watt Symposium IV (R. J. Knops, ed), Research Notes in Mathematics, Pitman, San Franscisco, 1979, 136-212.

7. W. Wasow, Asymptotic Expansions for Ordinary Differential Equations, Wiley Interscience, New York, 1965, 157ff.

Courant Institute, New York University, New York, NY 10012, USA 